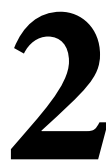

\title{
LAS TEORÍAS DE LA DESESCOLARIZACIÓN Y SU CONTINUIDAD EN LA PEDAGOGÍA LÍQUIDA DEL SIGLO XXI
}

\section{(THE DESCHOOLING THEORIES AND THEIR CONTINUITY IN THE LIQUID PEDAGOGY IN THE TWENTY FIRST CENTURY)}

Jon Igelmo Zaldívar

Universidad de Deusto

Xavier Laudo Castillo

Universidad de Valencia

DOI: $10.5944 / e d u c X X 1.17490$

\section{Cómo referenciar este artículo/How to reference this article:}

Igelmo Zaldívar, J. y Laudo Castillo, X. (2017). Las teorías de la desescolarización y su continuidad en la pedagogía líquida del siglo XXI. Educación XX1, 20(1), 37-56, doi: 10.5944/ educXX1.17490

Igelmo Zaldívar, J. \& Laudo Castillo, X. (2017). Las teorías de la desescolarización y su continuidad en la pedagogía líquida del siglo XXI. [The deschooling theories and their continuity in the liquid pedagogy in the twenty first century]. Educación XX1, 20(1), 37-56, doi: 10.5944/ educXX1.17490

\section{RESUMEN}

Este artículo parte de la existencia de un importante y creciente número de discursos y prácticas pedagógicas normativamente flexibles durante las dos primeras décadas del siglo XXI en Occidente. Se asocia este paradigma pedagógico emergente con la metáfora líquida (Bauman) y se resalta la aparición de procesos educativos diseñados específicamente para variar su forma y contenido en función de las circunstancias. Los autores exploran los orígenes del actual paradigma pedagógico líquido y exponen por qué constituye una continuidad en el tiempo con teorías anteriores, como las teorías de la desescolarización desarrolladas en los años sesenta y setenta. La metodología del estudio es la postsocial history (Joyce, Cabrera) que, a través del concepto de imaginario social (Taylor), permite estudiar las realidades discursivas emergentes que explican los cambios en las ideas, creencias y prácticas de los actores históricos. El estudio concluye que los discursos pedagógicos líquido y desescolarizador responden a la irrupción de un acontecimiento discursivo previo que resulta ser condición de posibilidad de ambos. 


\section{PALABRAS CLAVE}

Teoría de la educación; historia de la educación; historiografía; teorías de la desescolarización; pedagogía líquida; postmodernidad.

\section{ABSTRACT}

In this paper we take as a reference the reality of an important and growing amount of normative flexible pedagogical discourses and practices in the western world during the first two decades of the twenty first century. Understanding this novelty as an emergent pedagogical paradigm linked to the liquid metaphor (Bauman), we highlight the way in which new educative processes, specifically designed to change their shape and content depending on the circumstances, are arising. We aim to survey the origins of the currently liquid pedagogical paradigm and to display why this paradigm maintains a correlation of continuity with early theories, such as the deschooling theories developed in the 1960's and 1970's. The methodology selected to conduct this research is the post-social historiography (Joyce, Cabrera). This trend in historiography, using the concept of social imaginary (Taylor), allows us to study the emerging discursive realities that explain the changes within the ideas, beliefs and actions of the historical actors. The conclusion of this study underlines that liquid and deschooling pedagogical discourses respond to the emergence of a previous discursive event that is a condition of possibility for both.

\section{KEY WORDS}

Educational theory; educational history; historiography; deschooling theories; liquid pedagogy; postmodernity.

\section{INTRODUCCIÓN Y METODOLOGÍA}

Un número creciente de teorías y prácticas pedagógicas normativamente flexibles han aparecido en la última década (Bauman, 2005a). Asumimos esta novedad como la de un paradigma pedagógico emergente que puede comprenderse asociado a la metáfora de la liquidez: la pedagogía líquida. Así como el agua con sus continentes, los sujetos deben aprender a adaptarse constantemente a circunstancias contextuales cambiantes y órdenes morales relativos (Prats, 2005; Laudo, 2010). Este marco discursivo está cobijando un número creciente de redes ciudadanas desde las que se piensan nuevos itinerarios pedagógicos y enfoques educativos relativos a las tecnologías de la información y la comunicación, la crisis estructural del capitalismo y sus instituciones, o las alternativas a la educación institucio- 
nal tradicional —es el caso de las escuelas libres, los espacios pedagógicos autogestionados, o movimientos tan heterogéneos como el homeschooling, el unschooling, o el flexi-schooling-. Se trata de prácticas pedagógicas que, en su mayoría, con dificultad pueden encontrar referencias históricas dentro de la investigación educativa de carácter institucional que predomina en el contexto académico contemporáneo. A partir de la emergencia de este nuevo discurso pedagógico, inserto dentro del imaginario social postmoderno (Laudo, 2011), este trabajo plantea la necesidad de analizar, desde la teoría e historia de la educación, la genealogía discursiva del nuevo paradigma.

Como punto de partida asumimos que el conocimiento de lo que está en el presente necesita, inexorablemente, de un estudio detenido de aquello que lo precede en el pasado. La hipótesis que presentamos en este trabajo es que las prácticas características de la pedagogía líquida poseen una continuidad discursiva con las teorías desescolarizadoras. Una continuidad que puede ser estudiada a partir del análisis de tres características que se encuentran originalmente formuladas conjuntamente en las teorías de la desescolarización y que son compartidas por ambas ordenaciones pedagógicas: a) crítica al metarrelato de la educación, b) rechazo de la noción de progreso propia de la pedagogía moderna y c) cuestionamiento de la noción de emancipación individual y social como justificación y legitimación de los sistemas educativos. Lo que se muestra finalmente en este trabajo es que la emergencia del imaginario social postmoderno en la segunda mitad del siglo Xx puede ser comprendido como condición de posibilidad o marco epistemológico (Foucault, 1997) que habilita la articulación de estos tres elementos discursivos compartidos por las teorías desescolarizadoras y la pedagogía líquida.

Es importante subrayar que la relación que estableceremos entre las teorías de la desescolarización y el discurso pedagógico líquido no equivale a la que establece una línea recta con dos puntos fijos en el espacio. La ilación entre la corriente contra las instituciones educativas modernas y el imaginario pedagógico líquido, según lo que expondremos a continuación, queda mejor simbolizada si tomamos como referencia la representación matemática de la homotecia ${ }^{1}$. Desde esta perspectiva teórica, el imaginario social postmoderno surgido en la segunda mitad del siglo xx sería el equivalente a un punto fijo en el pasado, a partir del cual, a medida que fue extendiéndose, hizo posible que empezara a aparecer una crítica frontal al discurso pedagógico moderno y a la escuela como su máxima expresión institucional. Más tarde, y sobre el espacio que se fue abriendo a partir de ese punto original, se multiplicarían las distancias discursivas en los últimos cuarenta años hasta configurar el actual imaginario pedagógico líquido. Así, la emergencia de las teorías de la desescolarización y de la pedagogía líquida 
estarían directamente relacionadas con la mutación y el paso del imaginario social moderno al postmoderno, en lo que respecta a su dimensión pedagógica.

La metodología que nos permite abordar el estudio de las relaciones entre los fenómenos presentados es la que se propone desde la historia postsocial (Cabrera, 2004). Este enfoque centra su atención en el modo en que se articula y configura un determinado discurso, y no tanto en el impacto de las prácticas o ideas que se incluyen en él. Se asume que el discurso no es un medio de transmisión de los significados de la realidad por parte de sujetos racionales que lo utilizan a su antojo, sino un componente activo en el proceso de constitución de estos significados, y deviene así en una auténtica variable independiente que configura los procesos históricos (Cabrera, 2001, 52).

\section{LAS TEORÍAS DE LA DESESCOLARIZACIÓN EN LOS AÑOS 1960 Y 1970}

Para la teoría y la historia de la educación el enunciado de las teorías de la desescolarización aglutina al conjunto de críticas, hipótesis y alternativas expuestas en relación con el desempeño político, social, cultural y pedagógico de las instituciones educativas en la década de 1960 y 1970 (Igelmo Zaldívar, 2012). Los libros publicados por autores como Iván Illich, Everett Reimer, John Holt y Paul Goodman son, en buena medida, los que mejor representan no solo el contenido de la crítica desarrollada en este tiempo, sino también el tono en el que esta crítica fue planteada ${ }^{2}$. Visto desde la perspectiva histórica que dan los más de cuarenta años que han transcurrido desde la aparición de la desescolarización como corriente teórica, la principal novedad que para la pedagogía supuso el trabajo de esta generación de autores no reside en su método de estudio de las instituciones educativas modernas, ni en las alternativas pedagógicas que presentaron o siquiera en la crítica contra las escuelas presente en sus trabajos. Lo que cobra mayor importancia es la línea de sospecha abierta respecto a la contraproducente labor de las instituciones educativas modernas.

Gracias a las investigaciones desarrolladas en los últimos años se ha constatado que, aunque el fondo de la crítica a las instituciones educativas fue el elemento común compartido por los diferentes autores de esa corriente, existieron importantes matices en el enfoque de la crítica, así como en los objetivos que tenía el desarrollo de la misma. No es lo mismo, por tanto, la crítica que Illich planteó al modo en que la noción moderna de educación pervertía el aprendizaje - línea que desarrolló especialmente en los textos que publicó con posterioridad a su libro más afamado, esto es, 
La sociedad desescolarizada (1971) - (Bruno-Jofré e Igelmo Zaldívar, 2012; Cayley, 1992; Gabbard, 1994), que la necesidad detectada por Reimer en La escuela ha muerto (1973) de liberar a la educación del monopolio que sufría por parte de las escuelas y las instituciones educativas (Irizarry, 2009). De igual forma, tampoco puede equipararse el cuestionamiento de la labor de las escuelas como estructuras pedagógicas expuesto en los trabajos de John Holt (Farenga, 1998), con la crítica contracultural presentada por Paul Goodman en los libros que dedicó a analizar la cuestión de la educación y sus instituciones desde una perspectiva libertaria (Holowchak, 2010; Kaminsky, 2006).

Teniendo en mente lo expuesto en cuanto a las divergencias presentes en el pensamiento de esta generación de autores, es posible ubicar un aspecto común compartido: todos ellos detectaron la necesidad de explorar el descrédito que en la segunda mitad del siglo xx experimentaba el sistema institucional occidental moderno en su conjunto. Las escuelas, desde esta perspectiva, estudiadas como las instituciones encargadas de suministrar la educación, eran una pieza clave del entramado de instituciones que se presentaban entonces como una macroestructura capaz de garantizar los servicios que cubrían las necesidades de los individuos en sociedad. De ahí que, criticando a la escuela, y en esto el pensamiento de Illich, Reimer, Holt y Goodman converge, se pensaba que se podía combatir la parálisis psicológica, la castración social, el desarraigo cultural y la desigualdad económica con las que el mundo moderno y sus instituciones amenazaban a amplios sectores de la población. En última instancia lo que los autores de la desescolarización estaban poniendo en entredicho eran tres aspectos fundamentales del discurso educativo que una vez finalizada la Segunda Guerra Mundial habían posicionado a la escuela como la institución responsable de la educación de amplios sectores de la población por su propio bien: a) su estructuración como metarrelato moderno, b) la noción de progreso social que promovía y c) su contribución a la emancipación individual.

Para Holt, el hecho de que la educación fuera impuesta obligatoriamente y que las personas estuvieran forzadas a aprender determinados contenidos o habilidades era «una tiranía y un crimen contra la razón y el espíritu humano» (Holt, 1976, 222). Y es que la educación institucionalizada era percibida como un metarrelato que había nacido parapetado en el pensamiento escolástico y que había sufrido una importante renovación a la sombra del pensamiento científico al inicio del siglo xx. Pero a pesar de su renovación formal y técnica, la educación como metarrelato moderno se mostraba caduco y contraproducente en el contexto de expansión mundial de la escolarización obligatoria que se había puesto en marcha en la década de 1950. 
Este metarrelato de la educación institucional, además, según los teóricos de la desescolarización, tenía la misma presencia en los dos bloques que representaban los dos sistemas hegemónicos del momento, esto es: el capitalismo occidental y el socialismo. A ambos lados del telón de acero dirá Illich (1971, 113): «la escuela es una agencia de publicidad que te hace creer que necesitas el orden social tal y como es». El ideal de progreso, según fue analizado en libros como La sociedad desescolarizada, era el motor que movía tanto la maquinaria de los planes desarrollistas soviéticos como el crecimiento económico capitalista. El mito que sostenía la escolarización obligatoria se basaba en la falacia de que más años de escolarización traerían más progreso; más instituciones educativas traerían más elevados índices de desarrollo y crecimiento. Sin embargo, «el crecimiento concebido como el progreso basado en el eterno consumo sin límites nunca lleva a la madurez» (Ibíd, 43).

Asimismo, la crítica institucional planteada por los teóricos de la desescolarización ponía en entredicho la contribución que la educación podía realizar a la emancipación social e individual de los más desfavorecidos. En palabras de Goodman: «en nuestra sociedad, los jóvenes vivos y brillantes, con potencial de conocer, ideas nobles, esfuerzo honesto, y que alcanzan logros que valen la pena, son transformados en bípedos inválidos y cínicos, o en hombres decentes atrapados o resignados prematuramente, tanto fuera como dentro del sistema social» $(2012,22)$. Y es que una característica propia del pensamiento de los autores de la desescolarización es compartir la advertencia postmoderna de la falsedad de la idea de emancipación de cuño moderno; un tipo de ensoñamiento acrítico que destruye y pervierte la convivencia en sociedad, y que fue utilizado por los críticos de la educación y sus instituciones para articular una teoría de rechazo a cualquier institución que menoscabara la legítima libertad de los individuos. De ahí la necesidad planteada por todos estos autores de abrir los horizontes y expandir las posibilidades de aprendizaje más allá del cerrado mundo de las escuelas y su entramado institucional.

Según nuestro análisis, estas tres líneas de crítica articuladas conjuntamente en los trabajos presentados por los desescolarizadores suponen la apertura de nuevos márgenes no explorados dentro del imaginario pedagógico del momento. Aunque es cierto que con anterioridad se habían planteado en la historia críticas a las instituciones educativas en tanto que instituciones ${ }^{3}$, por primera vez una generación de autores, gracias al entorno discursivo postmoderno, puso en entredicho de modo coral el metarrelato emancipador y de progreso de la educación. Esto posibilitaba cuestionar el enunciado de la educación vigente hasta el momento y abrir los márgenes teóricos que permitían pensar en nuevas alternativas a los sistemas educativos existentes, así como tomar decisiones respecto a la orientación que la 
organización de la educación podía adoptar (Igelmo Zaldívar, 2013). De ahí la importancia de ubicar el lugar que corresponde a las teorías desescolarizadoras en la homotecia histórica que representa su continuidad discursiva con la pedagogía líquida postmoderna.

\section{EL DISCURSO PEDAGógICO LÍQUIDO EN EL SIGLO XXI}

Todavía en los años ochenta y noventa, la necesidad de fundamentos sólidos sobre los que apoyar las prácticas educativas siguió resquebrajándose. Este hecho sería estudiado desde la teoría educativa al observar que «un creciente número de educadores, encontrándose en problemas por los valores y asunciones modernistas, están girando hacia el postmodernismo en busca de ayuda para entender y articular sus preocupaciones» (Child, Williams, Birch y Boody, 1995, 167). El cambio de imaginario social y de contexto discursivo, de sólido a líquido en términos de Bauman, es lo que hizo que importantes autores afirmaran que lo que «hace "postmoderna" la teorización y la investigación educativas no es la disposición a renunciar al compromiso "moderno" con los valores emancipadores, sino la disposición a renunciar a la presunción de la Ilustración de que esos valores tengan que erigirse sobre unos "fundamentos" filosóficos» (Carr, 1995, 164). Se podrá seguir pensando la idea de emancipación con las herramientas que la crítica postmoderna nos dio para reconocer sus problemas y contradicciones (Bernal, 2011, 298), pero ya no con la ingenuidad dogmática del lema «la educación emancipa». Muchos educadores empezaron a plantear esquemas normativos que permitían mantener permanentemente abiertos espacios y tiempos donde otros significados pudieran ser pensados y articulados (Masschelein, 1998, 529-530). La vocación normativa de la pedagogía no se pierde con la crítica postmoderna, pero sí se ve necesariamente matizada (Laudo, 2011).

Este es el discurso de la pedagogía líquida, en el sentido de un conjunto coherente de categorías a través del cual los individuos significan y comprenden la realidad y llevan a cabo sus prácticas. Una definición podría ser esta: «el conjunto de disposiciones normativas sobre educación que propone unos medios que se adapten constantemente al contexto, cuyo fin es lograr que el educando encarne un modelo de persona adaptable a la incertidumbre y al cambio, y con habilidades hermenéuticas para interpretar la realidad sin referentes universales absolutos» (Laudo, 2010, 170). Como se puede ver, no deja de haber prescripción y normatividad, pues de algún modo debe haberlas para que haya intención educativa, pero estas son ahora entendidas cada vez más de un modo esencialmente adaptable y cambiante. Se toma como modelo educativo la metáfora de lo líquido: lo que cambia de forma adaptándose constantemente al entorno pero que, a 
su vez, como el agua sobre las rocas, también tiene fuerza transformadora y constituye una potencia de cambio.

Teóricas o prácticas, son diversas las corrientes y concreciones que se han ido elaborando bajo este paradigma en los últimos años. En algunos casos se han propuesto normatividades subordinadas a una «traducción cultural» constante, en función de cada contexto, y entendiendo esa labor como un proceso de adecuación y negociación cultural sin final, que no debe pretenderse como un momento intermedio para llegar a una meta ideal sino como un fin en sí mismo (Todd, 2009, 21-22). En otras coordenadas, autores como Vandenberg han postulado que el auténtico modo crítico en que debería hoy plantearse la educación no es decantarse ni por un modelo epistemológico (racionalismo, empirismo, escepticismo...) ni social (transformador, conservador...), sino adoptar una actitud de neutralidad inicial atenta y abierta a lo nuevo como mejor modo de aprender cualquier verdad y llevar a cabo cualquier educación: aceptando una combinación de las principales teorías del conocimiento, no como una síntesis sino como un "agregado flexible", asumiendo la posición de la "flexibilidad epistémica" (Vandenberg, 2009, 163). En un plano más vinculado con las propuestas pedagógicas de acción, esta articulación teórica coincide con numerosas prácticas pedagógicas implementadas por diferentes colectivos y sectores sociales.

\section{PROPUESTAS DE ENSEÑANZA-APRENDIZAJE NACIDAS A LA SOMBRA DE LAS NUEVAS TECNOLOGÍAS}

En concreto, a raíz de la consolidación de la versión 2.0 de la red de Internet un destacado número de propuestas pedagógicas están repensando las posibilidades de articular entornos de aprendizaje capaces de adaptarse a las necesidades, contextos culturales y gustos de cada individuo. El punto de partida es que las instituciones educativas modernas no están sabiendo responder al desafío presentado por la nueva generación de herramientas tecnológicas. Existe una opinión compartida según la cual el llamado cibermundo reclama una nueva concepción del aprendizaje (Bernal, 2009). $\mathrm{Al}$ tiempo, es cuestionado el relato emancipador educativo al marcar una gran distancia respecto a la certeza de que "la raíz de los problemas sociales resida en una falta de educación" (Downes, 2012, 541). Dentro de estos planteamientos pedagógicos se parte del supuesto de que la variedad de herramientas tecnológicas flexibles pueden ser articuladas de tal forma que cada persona pueda diseñar según su criterio su proceso de enseñanza-aprendizaje, ajustar sus objetivos formativos, organizar su red de socialización e incluso acotar el campo de acción donde se harán visibles los efectos de su aprendizaje. En esta línea se encuentran movimientos pedagógicos con un 
fuerte componente normativo, es el caso de la educación expandida (Díaz, 2011; Díaz y Freire, 2012), el conectivismo (Siemens, 2004, Downes, 2007; Sobrino, 2011), el procomún (Sguiglia, 2011), el aprendizaje ubicuo (Cope y Kalantzis, 2009; Burbules, 2012) o el aprendizaje invisible (Cobo y Moraveck, 2011) también proveniente de la metáfora líquida (Das, 2012). Cuaja la idea según la cual deja de ser necesaria para el educador o "diseñador" "la habilidad de señalar exactamente qué debe leerse y hacerse, en tanto que lo van definiendo [diseñador y estudiante] entre sî" (Siemens, 2011, 43).

\section{Alternativas pedagógicas críticas con los sistemas educativos oficiales al servicio de los sistemas políticos y económicos de Occidente}

Estas propuestas han alcanzado un desarrollo teórico destacado a partir de la reciente crisis financiera iniciada en el otoño de 2008 y parten de un rechazo frontal a la escuela como espacio de producción y consumo de conocimiento y aprendizaje. Desde estos movimientos se observa con desconfianza el vuelco operado en el seno del sistema educativo oficial que en las últimas décadas ha vinculado su desempeño con el desarrollo de las competencias demandadas por el mercado de trabajo, desatendiendo la formación integral de los individuos y la vinculación de estos con el medio ambiente (García Ajofrín, Miralles Lucena y Carbonell i Sebarroja, 2011). De ahí la reciente consolidación de alternativas vinculadas a movimientos ecológicos —es el caso de la ecopedagogía (Kahn, 2011)—, de iniciativas inspiradas en movimientos indigenistas - la Universidad de la Tierra en México es un buen ejemplo (Igelmo Zaldívar, 2009)_, el renacer de espacios formativos autogestionados - como ocurre con las escuelas libres anticapitalistas, siendo la escuela Paideia de Mérida una referencia internacional (Fremeaux y Jordan, 2012) - o los propios centros sociales ocupados. Espacios, todos ellos, que partiendo de un rechazo frontal del ideal de progreso y crecimiento propio del sistema capitalista de producción y consumo de bienes y servicios, buscan organizar procesos flexibles que permitan a cada individuo hacer de su proceso formativo un frente de lucha abierto contra el sistema capitalista y las instituciones educativas a su servicio.

\section{Redes ciudadanas que apuestan por la autogestión de los procesos formativos}

Aunque el rechazo a los sistemas oficiales predomina entre quienes desarrollan las propuestas de fundamento líquido, es importante mencionar que este no es un elemento necesario para la pedagogía líquida. El hecho que corrobora esta cuestión es la existencia de redes ciudadanas que sin desarrollar una crítica sistémica de las instituciones educativas oficiales apues- 
tan por la articulación de iniciativas que permitan la autogestión de los procesos formativos desmarcándose de una gestión heterónoma propia del metarrelato moderno. Así ocurre con muchas de las llamadas escuelas libres que carecen de una orientación política definida, las cuales son cada vez más numerosas y gozan de una mayor difusión dentro de las redes que tratan de organizar procesos flexibles y autogestionados con el objetivo de que cada individuo encuentre el sentido y significado de su proceso de aprendizaje. Los movimientos del homeschooling o sus variantes del unschooling y el flexischooling están en el origen de muchas de estas iniciativas (Elizalde, Urpí, Tejada, 2012; Goiria, 2012). Un ejemplo de ello lo encontramos en el ambiente educativo Ojo de agua, donde el agua y sus características líquidas representan en buena medida el modelo a seguir en el proceso de aprendizaje (Donadeu, 2012). Estas prácticas desarrollan la idea de autonomía en relación a la flexibilidad, y concitan cada vez más el interés de la comunidad educativa (la International Conference on Family Education Homeschooling de 2011 llevaba el significativo título de "Educational Innovation, ICT and flexischool"), y que están, cada vez más, siendo integradas entre el conjunto de posibilidades pedagógicas disponibles que son, en mayor o menor grado, socialmente aceptadas.

En definitiva, estas tres líneas de acción pedagógica ponen de manifiesto que los educadores postmodernos no se comprometen con teorías ni con actividades o articulaciones teóricas, sino solo con las creencias que subyacen a sus prácticas (Carr, 2006, 154-155); unas creencias que hoy son las que conforman el conjunto del imaginario social postmoderno. Se trata de una «nueva normatividad» que evita las decisiones directamente deducibles de una teoría, y cuya «emergente novedad radica en su orientación hacia la apertura y la indecidibilidad» (Van Goor, Heyting y Vreeke, 2004, 192). La pérdida del potencial prescriptivo y crítico del fundamento se concibe como una limitación que puede convertirse en una ventaja: «tomar esa carencia como punto de partida» (Ibíd., 186). La pedagogía líquida es, entonces, un discurso pedagógico dentro del imaginario social postmoderno que propone la transmisión de un modelo de mundo con referentes, verdades y realidades no absolutas sino relativas, no universales y atemporales sino contingentes e históricas. Un discurso en el que se configura como una certeza de primer orden educar según la norma de adaptarse constantemente al contexto como la mejor forma de preparar en un ambiente social y cultural incapacitado para articular metarrelatos sólidos. Se trata de una trama discursiva sobre la educación donde lo líquido y el cambio constante se postulan como valor normativo, en contraposición con los lineales planteamientos de la tecnología de la educación y las nociones de progreso y desarrollo como elementos clave en el camino de la emancipación. 


\section{EL IMAGINARIO SOCIAL POSTMODERNO: CONDICIÓN DE POSIBILIDAD DE LOS DISCURSOS DESESCOLARIZADORES Y DE LA PEDAGOGÍA LÍQUIDA}

Entendemos aquí por imaginario social la forma en que las personas «imaginan su existencia social, el tipo de relaciones que mantienen unas con otras, el tipo de cosas que ocurren entre ellas, las expectativas que se cumplen habitualmente y las imágenes e ideas normativas más profundas que subyacen a estas expectativas» (Taylor, 2002, 107). Es decir, se trata de un marco de referencia o espacio de pensamiento que delimita lo posible y lo imaginable, al tiempo que define por defecto lo que es imposible de imaginar. Dicho de otro modo, un imaginario social engloba «la concepción colectiva [common understanding] que hace posibles las prácticas comunes y un sentimiento ampliamente compartido de legitimidad» (Taylor, 2006, 37). De tal forma, al prefigurar las expectativas de los actores históricos, el imaginario social establece las condiciones de posibilidad de la acción. Siguiendo este enfoque, las prácticas de los sujetos históricos no estarían, en última instancia, determinadas por los hechos mismos, sino por la forma históricamente específica en que estos han sido dotados de significado mediante un imaginario social, mero entorno material o referente factual de dicha práctica, pero sin poder de determinación causal (Cabrera, 2003, 22-23). En este proceso, la significación que los individuos en sociedad dan a los hechos queda inserta en un espacio cognitivo cuya forma es consecuencia de la totalidad de los referentes teóricos que dan sentido a la existencia humana. De este modo, ese imaginario va modificando su forma y contenido en la medida que nuevos referentes teóricos son construidos sobre la base de las metáforas axiales sobre las que se fundamenta, a su vez, la experiencia existencial.

Aplicando la teoría que acabamos de exponer, tanto modernidad como postmodernidad, más allá del estéril debate nominalista, son conceptos abiertos que señalan entornos discursivos y que constituyen un conjunto de formas simbólicas a partir de las cuales se da significado y sentido a las teorías y prácticas de los actores sociales. En este sentido podemos comprender cómo el imaginario social moderno en Occidente constituyó un marco sólido en su fundamento y lineal en su pretensión de desarrollo, basado en el consenso normativo del triunfo de la razón para conseguir el progreso y perfección del futuro, con una objetivación de la realidad accesible mediante la ciencia, y donde la educación era percibida como la herramienta por excelencia para emancipar tanto individuos como sociedades. Este marco sostuvo los pilares de una estructura institucional que durante los dos últimos siglos se extendió por una gran parte de las regiones del mundo. Baste mencionar que la institucionalización de la educación, la salud o los medios de transporte, en su versión de servicios, se expandió históricamente 
al abrigo de este imaginario. Más tarde, en el tránsito hacia la postmodernidad, y según expuso Lyotard, entraría en crisis la credulidad respecto a estos metarrelatos legitimadores del saber, marcos discursivos de emancipación de la humanidad y de especulación y conocimiento científico de la realidad (Lyotard, 1994, 9-10, 63-73).

$\mathrm{Al}$ analizar desde una perspectiva histórica el caso del discurso educativo moderno, Daniel Tröhler señala que fue en pleno apogeo de la carrera espacial protagonizada por Estados Unidos y la Unión Soviética, simbolizado con el lanzamiento del Sputnik en 1957, cuando se «desencadenó la pedagogización de la guerra fría» (Tröhler, 2013, 231). En este proceso de pedagogización se constituyó una reconfiguración del lugar de la educación dentro de un imaginario social en el que el «desarrollo» se situaría como concepto clave capaz de servir de motor que pusiera en funcionamiento un nuevo marco discursivo para las políticas internacionales, la economía global y el progreso social (Sachs, 2010). Se comprendió entonces que «el motor necesitaba combustible y que ese combustible eran las personas educadas» (Tröhler, 2009, 7).

No obstante, como ya ha sido analizado por autores como Antonio Campillo, fue precisamente en el tiempo en que la pedagogización del mundo se posiciona como salvaguarda del progreso cuando comienza a ser observado con recelo el discurso del "progreso benefactor» (Campillo, 1985). Lo que nos lleva a ubicar con precisión en la década de los años cincuenta un primer nivel de configuración de las condiciones de posibilidades para la crítica al metarrelato educativo que en los años sesenta y setenta sería elaborado por los teóricos de la desescolarización. Y es que entonces comenzaban a asentarse las bases de la «sociedad moderna líquida»: aquella en la que «las condiciones sobre las que sus miembros actúan cambian más rápido de lo que tardan en consolidarse en hábitos y rutinas» (Bauman, 2005 b, 1). Una sociedad en la que habitan formas de vida, ideas y prácticas que se distancian del modelo ascendente que deja el rastro del progreso en la historia y que se caracteriza por la precariedad y las condiciones de incertidumbre constante. Unos enunciados que se convirtieron en supuestos intrínsecos de un imaginario social que, en la traducción pedagógica llevada a cabo por autores como Illich, Holt, Goodman y Reimer, hizo posibles y legítimos planteamientos educativos que en el paradigma anterior se considerarían contradicciones intolerables.

En consecuencia, el tiempo en que los críticos de la escolarización pusieron en marcha sus teorías podrían describirse como de transición entre Modernidad y Postmodernidad. Nos referimos a un momento de transición de imaginario social que deja sembrado el terreno para la formulación de una nueva crítica al discurso predominante. Un periodo de tiempo en el que 
las metáforas axiales que dan sentido a la existencia están siendo sustituidas, aunque ello no suponga, de momento, la imposibilidad de coexistencia de distintos imaginarios a la vez.

Recapitulando, consideramos que en los años sesenta y setenta del siglo XX, en líneas generales, las grandes y absolutas verdades, como las grandes y absolutas instituciones, y sobre todo estas porque legitimaban a aquellas, eran ya cuestionables desde el imaginario social postmoderno, aunque se encontrara en un estado todavía inicial. El declive de las instituciones como medios de emancipación empezaba a ser una idea compartida y naturalizada de forma cada vez más extendida. Existía la posibilidad de articular una teoría de la sospecha sobre los tres aspectos fundamentales del discurso educativo mencionados: su estructuración como metarrelato, la vinculación con el progreso social y la promoción de la emancipación del individuo. En consecuencia, la escuela, así como la educación provista a través de ella, podían ya impugnarse con un cierto apoyo social. Siendo esta una posibilidad de crítica que no podía ser pensada en la primera mitad del siglo Xx.

Del mismo modo ocurre con la pedagogía líquida. Exceptuando la solución concreta de acabar con las instituciones escolares, el conjunto de supuestos compartidos que la integran coinciden en gran medida con los que estaban en la base de las teorías desescolarizadoras. Entre ellas: el rechazo a la obligatoriedad de prescribir y aprender contenidos y habilidades rígidos, homogeneizantes y los mismos para todos en base a un metarrelato determinado; el desacuerdo con visiones unívocas de la sociedad y prácticas educativas reproductoras del orden social establecido fruto del progreso; o la necesidad de un profesor explicador y unidireccional para poder acceder al conocimiento emancipador. Es decir: aunque aparentemente la pedagogía líquida y las teorías desescolarizadoras parezcan propuestas pedagógicas inconexas o no directamente relacionadas, las dos son fruto del mismo imaginario social postmoderno, condición de posibilidad necesaria de ambas.

\section{CONCLUSIONES}

Entrado el siglo XXI es un hecho que las personas buscan aprender más allá de las instituciones responsables de la formación de los individuos. De igual forma que es evidente que para disfrutar del arte, hablar nuevos idiomas, tocar un instrumento, cultivar una huerta, desarrollar técnicas de construcción ecológicas o estudiar historia, los individuos tienen a su disposición no solo las tecnologías en red de la información y la comunicación, sino también la posibilidad de tomar parte en colectivos ciudadanos que les permiten acceso directo, sencillo y práctico al conocimiento. En cierto modo, el descrédito contemporáneo a las instituciones educativas está estrechamente 
relacionado con la certeza postmoderna de que los espacios educativos formales sirven principalmente solo para acceder (aunque ya cada vez con más dificultad) un título que pueda abrir puertas en el mundo laboral. La labor tradicional de estos espacios como guardianes, generadores o transmisores del conocimiento se ha ido diluyendo en el imaginario pedagógico líquido. En el debate pedagógico actual los medios y métodos de enseñanza tradicionales encuentran cada vez más críticas y alternativas filosófico-pedagógicas (Laudo, 2013; Vansieleghem, N. y Masschelein, 2012; Dobson, 2012).

Hasta el momento, los estudios existentes sobre la crítica de los años sesenta y setenta a los sistemas educativos institucionales apenas habían superado la enumeración de autores, argumentos y experiencias educativas alternativas llevadas a cabo, pretendiendo explicar en qué grado la pedagogía de cada momento ha ido encontrando respuesta a los interrogantes planteados contra las instituciones escolares. En cambio, nosotros hemos querido explorar cual es su relación discursiva con las ideas básicas de un paradigma pedagógico emergente como el de la pedagogía líquida. En consecuencia, aplicando la metodología de la historia postsocial se puede afirmar que las teorías de la desescolarización son un acontecimiento clave para el estudio de la articulación y configuración del discurso propio de la pedagogía líquida. Su crítica al metarrelato educativo, el cuestionamiento de la noción de progreso ligada a la expansión escolar y el rechazo de la promesa propia del discurso pedagógico formal, son tres elementos que relacionan en el tiempo histórico a las teorías de la desescolarización y la pedagogía líquida. Por eso, a pesar del desigual impacto que la corriente de la desescolarización tuvo en los años ochenta y noventa para la comunidad pedagógica internacional, la vigencia y validez de ese discurso para las nuevas articulaciones teóricas y prácticas de argumentación líquida y postmoderna puede observarse ahora con más nitidez.

La crítica al discurso educativo moderno pensada por los teóricos de la desescolarización tiene hoy un renovado sentido, y este se hace más evidente en la medida en que el discurso pedagógico líquido sigue ganando espacio dentro del imaginario social de Occidente. La pedagogía líquida y las teorías desescolarizadoras comparten un mismo origen histórico y un mismo marco epistemológico, y es necesario tomarlos en cuenta para poder comprender el pasado, el presente y el futuro de cualquiera de ellas.

\section{NOTAS}

1 La homotecia es una transformación geométrica en la que, a partir de un punto fijo, multiplica todas las distancias por un mismo factor. Los fundamentos teóricos de este tipo de herramientas en la investigación histórica, tal y como lo han discutido y formulado Bonneuil (2010), Aubin y Dalmecio (2002) y McNeill (1998), se basan en la capacidad simbólica de 
representación que ofrece la relación entre la teoría de la historia y las propiedades matemáticas de la continuidad y la conexión.

2 Aunque es cierto que estos cuatro autores son los que con sus tesis mejor representan las conocidas como teorías de la desescolarización, se tiene presente que hubo muchos otros autores que aportaron importantes matices a esta corriente de pensamiento. Los volúmenes editados por el Centro Intercultural de Documentación de Cuernavaca (CIDOC) en relación al seminario "Alternatives in Education" son la fuente histórica que permite un mejor acercamiento a quienes junto con Illich, Reimer, Goodman y Holt participaron del debate abierto en este espacio de discusión, es el caso de: Joel H. Spring, Jordan Bishop, Didier Piveteau, Michel Debeauvais, Gerald Kamber, Peter Johnson, Paul Bady, John Ohliger, Maxine Greene, Samuel Bowles, Ian Lister, Dennis Sullivan (CIDOC, 1972a, 1972b).

3 En el campo de la teoría y la historia de la educación se han realizado estudios en relación a las diferentes críticas articuladas en distintos momentos históricos contra las instituciones educativas y su desempeño pedagógico y social. En este sentido es importante mencionar que ya en el siglo XIX se plantearon importantes cuestionamientos sobre la estructura y el desempeño de los sistema escolares, tal fue el caso de trabajo publicado por pensadores como William Godwin (Manly, 2012), Joseph Jacotot (Laudo, 2013; Rancière, 2010) y Liev Tolstói (Murphy, 1992). 


\section{REFERENCIAS BIBLIOGRÁFICAS}

Aubin, D. y Dalmecio, A. D. (2002). Writing the history of Dynamical Systems and Chaos: Longue Durée and Revolution, Disciplines and Cultures. Historia Mathematica, 29(3), 273-339.

Bauman, Z. (2005a). Education in Liquid Modernity. Review of Education, Pedagogy, and Cultural Studies, 27 (4), 303-317.

Bauman, Z. (2005b). Liquid Life. Cambridge: Polity Press.

Bernal, A. (2009). Cibermundo y educación. Bosquejo de un nuevo marco formativo en contextos postmodernos. Teoría de la educación, 21(1), 71-102.

Bernal, A. (2011). Postmodernización y educación. Notas para el debate de una narrativa pedagógica centrada en la identidad. Educación XX1, 14(2), 283-300.

Bonneuil, N. (2010). The Mathematics of Time in History. History and Theory, 49(4), 28-49.

Bruno-Jofré, R. \& Igelmo Zaldívar, J. (2012). Ivan Illich's Late Critique of Deschooling Society: "I Was Largely Barking up to the Wrong Tree". Educational Theory, 62(5), 573-592.

Burbules, N. (2012). Ubiquitous Learning and the Future of Teaching. Encounters on Education, 13, 3-14.

Cabrera, M.Á. (2001). Historia, lenguaje y teoría de la sociedad. Madrid: Cátedra.

Cabrera, M.Á. (2003). La crisis de lo social y su repercusión sobre los estudios históricos. Pasado y Memoria, Revista de Historia Contemporánea, 2, 5-35.

Cabrera, M.Á (2004). Postsocial History: An Introduction. Lanham: Lexington Books.
Campillo, A. (1985). Adiós al progreso. Una meditación sobre la Historia, Barcelona: Anagrama.

Carr, W. (1995). Una teoría para la educación. Hacia una investigación educativa crítica. Madrid: Morata.

Carr, W. (2006). Education Without Theory. British Journal of Educational Studies, 54(2), 136-159.

Cayley, D. (1992). Iván Illich in Conversation. Toronto: Anansi.

Child, M., Williams, D., Birch, A. \& Boody, R. (1995). Autonomy or Heteronomy? Levinas's Challenge to Modernism and Postmodernism. Educational Theory, 45(2), 167-189.

CIDOC (1972a). CIDOC documenta Alternatives in Education. July 1970-June 1971, v. 1 y 2. CIDOC Cuaderno no. 7576. Cuernavaca: Centro Intercultural de Documentación.

CIDOC (1972b). CIDOC Documenta Alternatives in Education. July 1971-June 1972, v. 1 y 2. CIDOC Cuaderno no. 77 78. Cuernavaca: Centro Intercultural de Documentación.

Cobo, C. y Moravec, J. W. (2011). Aprendizaje invisible. Hacia una nueva ecología de la educación. Barcelona: Publicacions i Edicions de la Universitat de Barcelona.

Cope, B. \& Kalantzis, M. (2009). Ubiquitous Learning. Urbana: University of Illinois.

Das, S. (2012). On two metaphors for pedagogy and creativity in the digital era: liquid and solid learning. Innovations in Education and Teaching International, 49(2), 183-193.

Díaz, R. (2011). ¿Qué escuela es imposible imaginar y de qué imposibilidad se 
trata?: apuntes para la investigación de una educación expandida. Sevilla, Universidad de Sevilla. Recuperado de: http://goo.gl/XemlgK

Díaz, R. y Freire, J. (ed.) (2012). Educación expandida. Sevilla: Zemos 98 y Universidad Internacional de Andalucía.

Donadeu, M. (2012). L'educació lliure. Una mirada a les experiències educatives alternatives. Girona: Universitat de Girona.

Downes, S. (2007). An Introduction to Connective Knowledge, en T. Hug (ed.) Media, Knowledge \& Education - Exploring new Spaces, Relations and Dynamics in Digital Media Ecologies. Recuperado de: http://goo.gl/CeP5or

Downes, S. (2012). Connectivism and Connective Knowledge. Recuperado de: http://goo.gl/hg6QY

Elizalde, M. A.; Urpí, C.; Tejada, M. (2012). Diversidad, participación y calidad educativas: necesidades de Homeschooling. Estudios Sobre Educación, 22, 55-72.

Farenga, P. (1998). Homeschooling: Creating Alternatives to Education. Bulletin of Science, Technology \& Society, 18(2), 127-133.

Foucault, M. (1997). Arqueología del saber. Madrid: Siglo XXI.

Fremeaux, I. \& Jordan, J. (2012). Anarchist Pedagogy in Action: Paideia, Escuela Libre. En R.H. Haworth Anarchist Pedagogies. Collective Actions, Theories, and Critical Reflections on Education. Estados Unidos: PM Press.

Gabbard, D.A. (1994). Ivan Illich, Postmodernism, and The Eco-crisis: Reintroducing a "Wild" Dicourse. Educational Theory, 44(3), 173-187.
Dobson, S. (2012). The Pedagogue as Translator in the Classroom. Journal of Philosophy of Education, 46(2), 271286.

García Ajofrín, L., Miralles Lucena, R, y Carbonell i Sebarroja, J. (2011). El movimiento del 15-m. Un aprendizaje muy democrático. Cuadernos de pedagogía, 414, 8-17.

Goiria, M. (2012) La flexibilización educativa o lo mejor de los mundos (entre la escolarización y el homeschooling). Estudios Sobre Educación, 22, 37-54.

Goodman, P. (2012). Growing up Absurd. Nueva York: New York Review of Books.

Hollowchak, M.A. (2010). Paul Goodman redux: education as apprenticed anarchism. Ethics and Education. 5(5), 217-232.

Holt, J. (1976). Instead of Education: Ways to Help People to Think Better. Nueva York: Dutto.

Igelmo Zaldívar, J. (2009). La Universidad de la Tierra en México. Una propuesta de aprendizaje convivencial, en J.L. Hernández Huerta, L. Sánchez Blanco, e I. Sánchez Miranda (ed.) Temas y perspectivas sobre educación. La infancia ayer y hoy. Vol 1. (pp. 285298). Salamanca: Globalia.

Igelmo Zaldívar, J. (2012). Las teorías de la desescolarización; cuarenta años de perspectiva histórica. Historia social y de la educación, 1(1), 28-57. Recuperado de: http://goo.gl/kjuCp1

Igelmo Zaldívar, J. (2013). Paulo Freire y John Holt: de la educación liberadora a la libertad más allá de la educación. Educació i Història, 21, 13-35. Recuperado de: http://goo.gl/Pe9K7y

Illich, I. (1971). Deschooling Society. Nueva York: Harper and Row. 
Irizarry, R. (2009). Everett Reimer, de la ingeniería social en Puerto Rico a la deconstrucción y la utopia. La Torre: Revista de la Univerisdad de Puerto Rico, 14(53), 261-278.

Kahn, R. (2011). Ecopedagogy: educating for sustainability in schools and society. Estados Unidos: Routedge.

Kaminsky, J.S. (2006). Paul Goodman, 30 Years Later: Growing Up Absurd; Compulsory Mis-education, and The Community of Schoolar; and The New Reformation- A Retrospective. Teachers College Record, 108(7), 13391361.

Laudo, X. (2010). La pedagogía líquida. Fuentes contextuales y doctrinales. Barcelona: Universidad de Barcelona. Recuperado de: http://www.tdx.cat/ handle/10803/2958

Laudo, X. (2011). La hipótesis de la pedagogía postmoderna. Educación, verdad y relativismo. Teoría de la Educación. Revista Interuniversitaria, 23(2), 45-68. Recuperado de: http:// goo.gl/mCCTga

Laudo, X. (2013). Educación y emancipación: de la experiencia de Jacotot a la expectativa de Rancière. Educació $i$ Història, 21, 67-87. Recuperado de: http://goo.gl/xLpQrR

Lyotard, J. F. (1994). La condición postmoderna. Madrid: Cátedra.

Manly, S. (2012). William Godwin's "School of Morality". Wordworth Circle, 43(3), 135-142.

Masschelein, J. (1998). How to imagine something exterior to the System: Critical Education as problematization. Educational Theory, 48 (4), 521-530.

McNeill, W. H. (1998). History and the Scientific Worldview. History and Theory, 37(1), 1-13.
Murphy, D. (1992) Tolstoi and Education. Ireland: Irish Academic Press.

Prats, E. (2005). Pedagogia líquida per a una educació sòlida: a propòsit de Bauman. Temps d'Educació, 28, 37-324.

Rancière, J. (2010). El maestro ignorante. Cinco lecciones sobre la emancipación intelectual. Barcelona: Laertes.

Reimer. E. (1973). La escuela ha muerto. Alternativas en materia de educación. Barcelona: Barral.

Sachs, W. (ed.) (2010). The Development Dictionary. A Guide to Knowledge the Power. New York: Zed Books.

Sguiglia, N. (2011). Libertad, autonomía y procomún. Movimientos urbanos en la era de la precariedad, en A. Calle Collado (Ed.) Democracia radical: entre vínculos y utopías. Madrid: Icaria.

Siemens, G. (2011). Entrevista a George Siemens desarrollador del conectivismo. Revista mexicana de bachillerato a distancia, 6, 41-47. Recuperado de: http://goo.gl/09W5Ui

Siemens, G. (2004). Connectivism: A Learning Theory for the Digital Age. Recuperado de: http://goo.gl/uU4QI

Sobrino, Á. (2011). Proceso de enseñanza-aprendizaje 2.0. Valoración del conectivismo como teoría de aprendizaje post-constructivismo. Estudios sobre educación, 20, 117-140.

Taylor, C. (2002). Modern social imaginaries. Public Culture, 14(1), 91-124.

Taylor, C. (2006). Imaginarios Sociales Modernos. Barcelona: Paidós.

Todd, S. (2009). Universality and the Daunting Task of Cultural Translation. Journal of Philosophy of Education, 43(1), 1-29.

Tröhler, D. (2013). Los lenguajes de la educación. Barcelona: Octaedro. 
Tröhler, D. (2009). Armonizar el globo educativo. Política mundial, rasgos culturales y los desafíos a la investigación educativa. Profesorado. Revista de currículum y formación del profesorado, 13(2), 1-14.

Van Goor, R., Heyting, F. \& Vreeke, G-J. (2004). Beyond Fundations: Signs of a New Normativity in Philosophy of Education. Educational Theory, 54(2), 173-192.
Vandenberg, D. (2009). Critical thinking about truth in teaching: The epistemic ethos. Educational Philosophy and Theory, 41(2), 155-165.

Vansieleghem, N. \& Masschelein, J. (2012). Education as Invitation to Speak: On the Teacher Who Does Not Speak. Journal of Philosophy of Education, 46(1), 85-99. 


\section{PERFIL ACADÉMICO Y PROFESIONAL DE LOS AUTORES}

Jon Igelmo Zaldívar. Investigador postdoctoral de la Universidad de Deusto con financiación del Programa de Formación de Personal Investigador del Gobierno Vasco. Es miembro del grupo INNOVA (Universidad de Deusto), del grupo Cultura Cívica y Políticas Educativas (Universidad Complutense de Madrid) y del Theory and History of Education International Research Group (Queen's University, Canada). En 2015 ha sido seleccionado para una ayuda Juan de la Cierva-incorporación del Ministerio de Economía y Competitividad.

Xavier Laudo Castillo. Profesor Ayudante Doctor del departamento de Educación Comparada e Historia de la Educación de la Universidad de Valencia. Es miembro del Grupo de Investigación en Pensamiento Pedagógico y Social (UB-Generalitat de Catalunya) y Premio Extraordinario de Doctorado por la Universidad de Barcelona.

Dirección de los autores: Jon Igelmo Zaldívar

Facultad de Psicología y Educación, despacho 234 (bis)

Universidad de Deusto

Avenida de las Universidades, 24

48007, Bilbao, Bizkaia

E-mail: jigelmoza@deusto.es

Xavier Laudo Castillo

Universitat de València

Facultat de Filosofia i Ciències de l'Educació Dept. d'Educació Comparada i Història de l'Educació

Av. Blasco Ibáñez, 30

46010 - València

E-mail: Xavier.Laudo@uv.es

Fecha Recepción del Artículo: 22. Enero. 2014

Fecha modificación Artículo: 22. Abril. 2014

Fecha Aceptación del Artículo: 28. Abril. 2014

Fecha Revisión para Publicación: 06. Julio. 2016 